\title{
Emoções manifestas por adolescentes escolares na pandemia COVID-19
}

\author{
Emotions manifested by school adolescents in the COVID-19 pandemic \\ Emociones manifestadas por adolescentes escolares en la pandemia COVID-19
}

Recebido: 21/02/2021 | Revisado: 28/02/2021 | Aceito: 17/03/2021 | Publicado: 20/03/2021

\author{
Aline Dias Gomes \\ ORCID: https://orcid.org/0000-0002-7761-1948 \\ Universidade Federal Fluminense, Brasil \\ Fundação Municipal de Saúde. Brasil \\ E-mail: alinedg4@gmail.com \\ Claudia Mara de Melo Tavares \\ ORCID: https://orcid.org/0000-0002-8416-6272 \\ Universidade Federal Fluminense, Brasil \\ E-mail: claudiatavares@id.uff.br \\ José Carlos Carvalho \\ ORCID: https://orcid.org/0000-0002-8391-8647 \\ Escola de Enfermagem do Porto, Portugal \\ E-mail: zecarlos@esenf.pt \\ Maryana Tavares e Souza \\ ORCID: https://orcid.org/0000-0003-3693-3673 \\ Universidade Federal Fluminense, Brasil \\ E-mail: maryanatavs@gmail.com \\ Marilei de Melo Tavares e Souza \\ ORCID: https://orcid.org/0000-0002-3276-0026 \\ Universidade de Vassouras, Brasil \\ E-mail:marileimts@hotmail.com
}

\section{Resumo}

Diante da pandemia pela COVID-19 a principal ocupação dos adolescentes, os estudos, sofreu uma mudança abrupta com o isolamento social e a suspensão das aulas presenciais podendo acarretar sofrimento emocional a esse grupo. Objetivo: Identificar manifestações emocionais dos adolescentes escolares diante da pandemia por COVID 19 e estratégias de manejo emocional por eles adotadas. Método: Estudo exploratório-descritivo, realizado a partir da articulação Universidade-Atenção Básica-Escola. Foi enviado um questionário do Google Forms, via plataforma escolar aos adolescentes estudantes do ensino médio de uma escola pública da cidade de Niterói-RJ. Após um mês de disponibilização do questionário 15 estudantes responderam. Para análise dos dados calcularam-se as estatísticas apropriadas e as frequências absolutas e percentuais. O estudo foi aprovado pelo Comitê de Ética e Pesquisa - Parecer No 3.671.594. Resultados: As principais emoções expressas pelos adolescentes escolares diante da Pandemia foram cansaço $(63,6 \%)$, tristeza (45,5\%), saudades do colégio e dos amigos (45,5\%), falta das aulas presenciais $(18,2 \%)$, indiferença $(27,3 \%)$ e depressão $(9,1 \%)$. Emoções positivas como alegria $(27,3 \%)$ também foram identificadas. Todos informam que tiveram parentes, amigos, vizinhos ou conhecidos infectados e $36,4 \%$ conheceram alguém que faleceu. Os dados evidenciaram que todos adolescentes estão conectados a uma rede virtual e apontam como estratégias de manejo emocional implementadas por eles mesmos: música $(63,6 \%)$, redes sociais $(54,5 \%)$, bate papo com amigos $(45,5 \%)$, jogos/games (36,4\%), igreja e namoro (27,3\%), esporte, escola, filmes e séries $(9,1 \%)$. Conclusões: Os adolescentes escolares estão vivenciando transtornos emocionais em decorrência da pandemia e têm implementado por conta própria estratégias de manejo emocional.

Palavras-chave: Adolescentes; Saúde Mental; Saúde Escolar; COVID-19.

\begin{abstract}
Faced with the pandemic by COVID-19, the main occupation of adolescents, studies, has undergone an abrupt change with social isolation and the suspension of face-to-face classes, which can cause emotional suffering for this group. Objective: To identify emotional manifestations of school adolescents in the face of the COVID pandemic 19 and emotional management strategies adopted by them. Method: Exploratory-descriptive study, carried out from the University-Primary Care-School articulation. A Google Forms questionnaire was sent, via the school platform, to adolescent high school students from a public school in the city of Niterói-RJ. After a month of making the questionnaire available, 15 students answered. For data analysis, the appropriate statistics and absolute and percentage frequencies were calculated. The study was approved by the Ethics and Research Committee - Opinion No. 3,671,594. Results: The main emotions expressed by school adolescents in the face of Pandemic were tiredness $(63.6 \%)$, sadness (45.5\%), longing for school and friends (45.5\%), lack of face-to-face classes (18.2\%), indifference (27.3\%) and depression $(9.1 \%)$. Positive emotions such as joy $(27.3 \%)$ were also identified. Everyone reports that they had infected relatives, friends, neighbors or acquaintances and $36.4 \%$ knew someone who died. The data showed that all
\end{abstract}


adolescents are connected to a virtual network and point out as emotional management strategies implemented by themselves: music (63.6\%), social networks (54.5\%), chatting with friends (45.5\%), games / games (36.4\%), church and dating (27.3\%), sports, school, films and series (9.1\%). Conclusions: School adolescents are experiencing emotional disorders as a result of the pandemic and have implemented emotional management strategies on their own.

Keywords: Keywords: Adolescents; Mental health; School Health; COVID-19.

\section{Resumen}

Frente a la pandemia por COVID-19, la principal ocupación de los adolescentes, los estudios, ha sufrido un cambio abrupto con el aislamiento social y la suspensión de las clases presenciales, lo que puede ocasionar sufrimiento emocional a este colectivo. Objetivo: Identificar las manifestaciones emocionales de los adolescentes escolares frente a la pandemia de COVID 19 y las estrategias de manejo emocional adoptadas por ellos. Método: Estudio exploratoriodescriptivo, realizado a partir de la articulación Universidad-Atención Primaria-Escuela. Se envió un cuestionario de Google Forms, a través de la plataforma escolar, a estudiantes adolescentes de secundaria de una escuela pública de la ciudad de Niterói-RJ. Después de un mes de tener disponible el cuestionario, 15 estudiantes respondieron. Para el análisis de los datos, se calcularon las estadísticas apropiadas y las frecuencias absolutas y porcentuales. El estudio fue aprobado por el Comité de Ética e Investigación - Opinión No. 3.671.594. Resultados: Las principales emociones expresadas por los adolescentes escolares ante la pandemia fueron cansancio (63,6\%), tristeza (45,5\%), añoranza por la escuela y amigos $(45,5 \%)$, falta de clases presenciales $(18,2 \%)$, indiferencia $(27,3 \%)$ y depresión $(9,1 \%)$. También se identificaron emociones positivas como la alegría $(27,3 \%)$. Todo el mundo informa que había contagiado a familiares, amigos, vecinos o conocidos y el 36,4\% conocía a alguien que falleció. Los datos mostraron que todos los adolescentes están conectados a una red virtual y señalan como estrategias de manejo emocional implementadas por ellos mismos: música $(63,6 \%)$, redes sociales $(54,5 \%)$, chat con amigos $(45,5 \%)$, juegos / juegos $(36,4 \%)$.), iglesia y citas $(27,3 \%)$, deportes, escuela, películas y series $(9,1 \%)$. Conclusiones: Los adolescentes escolares están experimentando trastornos emocionales debido a la pandemia y han implementado estrategias de manejo emocional por su cuenta.

Palabras clave: Adolescentes; Salud mental; Salud escolar; COVID-19.

\section{Introdução}

A adolescência é uma fase de grandes transformações que podem afetar a saúde emocional dos jovens. De modo geral eles apresentam uma boa saúde física e mental, entretanto, a exposição a fatores sociais graves como violência, pobreza ou abandono pode colocá-los em situação de vulnerabilidade. Atividades de promoção da saúde mental ajudam os adolescentes a lidarem com suas emoções, a desenvolverem habilidades interpessoais para auxiliar na resolução de problemas e a construir ambientes de apoio na família, na escola ou na comunidade.

Em dezembro de 2019 um novo fator de vulnerabilidade surge para agravar ou, se somar aos acima expostos, a pandemia pelo coronavirus (SARS-CoV-2). Diante da pandemia, diversas ações foram adotadas pelos governantes dos países afetados para tentar conter o avanço da doença ou diminuir a velocidade de novos casos utilizando a quarentena e o isolamento social como as principais medidas.

Com o avanço da COVID-19, as medidas de isolamento, fechamento de escolas, de universidades, de grande parte do comércio e a quarentena passaram a ser adotadas no Brasil a partir de meados do mês de março de 2020 impondo a todos uma grande mudança nos hábitos de vida. As aulas presenciais foram substituídas por aulas em meios digitais após a publicação feita pelo Ministério da Educação da Portaria nº 343, de 17 de março de 2020 (BRASIL, 2020).

O método mais eficaz, disponível até o momento, para o controle da doença - o distanciamento social traz sequielas à saúde mental da população como: estresse pós-traumático, confusão, raiva, medo de infecção, frustração, tédio, suprimentos inadequados, informações inadequadas, perda financeira e estigma que podem ser maiores que o número de mortes (Brooks et al., 2020).

Durante o confinamento da quarentena os níveis de ansiedade, depressão e estresse aumentam gerando um impacto psicológico negativo nos estudantes. Torna-se imprescindível dar continuidade às relações sociais, estimular a solidariedade e a resiliência nesse período (MAIA\& DIAS, 2020).

Pelo fato da pandemia da COVID-19 ser um fenômeno recente existem poucos estudos que abordam seus impactos na saúde mental, mas repercussões negativas importantes são apontadas como: medo, estresse, mudança das relações 
interpessoais, insegurança quanto à subsistência familiar (Schmidt et al., 2020). Todos estes fatores aumentam a vulnerabilidade de crianças e adolescentes.

Características típicas da adolescência como condutas oposicionistas e retraimento podem se exacerbar nesse período. Um modo de amenizar tais características é o encontro com os pares sendo a escola o local onde eles ocorrem normalmente. O fechamento de escolas iniciou em março de 2020 e permanece até o momento atual, fato que amplia o risco de abandono escolar permanente, principalmente por parte de crianças e adolescentes em situação de vulnerabilidade. Além disso, o fechamento das escolas interrompe atividades extracurriculares, programas recreativos e acesso a outros serviços básicos importantes (UNICEF, 2020).

A escola pública atende a maioria das crianças e adolescentes brasileiras e exerce um papel muito além da aprendizagem, o enfrentamento das desigualdades sociais (Júnior, 2020). É na escola que a maioria dos abusos, agressões e oportunidades são desveladas.

Não podemos esquecer que saúde física e saúde mental andam juntas. Fatores como o confinamento prolongado, a diminuição de atividade física, a falta de contato pessoal com os colegas de classe, o medo e a incerteza do futuro, atingem a saúde mental de boa parte dos estudantes e de suas famílias.

Educadores, profissionais de saúde e pais estão sendo desafiados a lidar com as emoções dos adolescentes em função das mudanças ocorridas em suas vidas, principalmente no que diz respeito ao distanciamento social do ambiente escolar decorrente da Pandemia COVID19.

Diante dessa nova situação, os adolescentes precisam de estratégias de ação para gerenciar suas emoções, minimizando o impacto das atuais circunstâncias na sua saúde mental.

O presente estudo teve por objetivo identificar as emoções expressas por adolescentes escolares diante do distanciamento social decorrente da Pandemia por COVID 19 e discutir as estratégias adotadas para manejar suas emoções.

\section{Metodologia}

Trata-se de uma pesquisa descritivo-exploratória, qualitativa, utilizando o método de estudo de caso (EC) sobre a promoção da saúde mental dos adolescentes que se encontram em distanciamento social e afastamento da escola em decorrência da pandemia por COVID-19 (Pereira, 2018).

Em função da impossibilidade da obtenção de dados por meio de entrevista presencial decorrente da Pandemia, foi elaborado um instrumento de coleta de dados enviado para a direção da escola e analisado em reunião do colegiado. Após a autorização da escola, da aquiescência dos pais e aprovação do Comitê de Ética em Pesquisa, o questionário de coleta de dados via Google Forms, foi inserido na Plataforma online do colégio, sendo os adolescentes estudantes do ensino médio convidados a respondê-lo.

O questionário constou de uma breve apresentação da pesquisa, um convite para a participação, o Termo de Livre Consentimento Esclarecido (TCLE) e um link para acesso ao questionário. O questionário continha como questões principais: as emoções, o enfrentamento do distanciamento social, acesso dos adolescentes aos serviços de saúde e medidas de manejo emocional.

Os participantes da pesquisa foram os adolescentes escolares matriculados no ensino médio de uma escola pública do município de Niterói-RJ, Brasil.

Após aproximadamente 30 dias da disponibilidade do questionário na Plataforma da Escola, 15 alunos responderam à pesquisa, embora nesse período a escola tenha contabilizado 77 acessos à Plataforma.

Para análise dos dados calcularam-se as estatísticas apropriadas e as frequências absolutas e percentuais. O estudo foi aprovado pelo Comitê de Ética e Pesquisa - Parecer No 3.671.594. 


\section{Resultados}

Um total de 15 adolescentes escolares participou desta pesquisa. A faixa etária variou de 17 a 18 anos; 63,3\% do sexo feminino e $36,4 \%$ do sexo masculino; $36,4 \%$ estão no $1^{\circ}$ ano do ensino médio, $36,4 \%$ no $3^{\circ}$ ano e $27,3 \%$ no $2^{\circ}$ ano. Todos informaram ter acesso a internet.

Todos informam que tem algum membro da família que não parou de trabalhar durante a pandemia e que os hábitos da casa mudaram com a COVID-19. Quando questionados se eles e sua família estão em isolamento social, 54,5\% informaram que sim, 45,5\% disseram estar em isolamento parcial e nenhum deles não estava fazendo isolamento social.

A COVID-19 é uma realidade bem próxima para os entrevistados visto que 90,9\% tiveram parentes, amigos, vizinhos ou conhecidos infectados e 36,4\% conheceram alguém que faleceu. Nenhum deles teve a doença e apenas $9,1 \%$ informaram que não conheciam ninguém que contraíra a enfermidade.

As emoções expressas relacionadas ao momento atual de Pandemia foram: tristeza (45,5\%); cansaço ou desânimo (66,6\%); pesar pelo afastamento da escola (45,5\%), dos amigos (45,5\%) e das aulas presenciais $(18,2 \%)$; indiferença $(27,3 \%)$; depressão $(9,1 \%)$; alegria e outras emoções positivas $(27,3 \%)$.

Em relação aos profissionais de saúde, $54,5 \%$ afirmaram que se sentem confortáveis para falar sobre suas necessidades emocionais com um e 45,5\% não se sentem confortáveis. Mas ao serem questionados se quando sentissem tristes, angustiados procurariam um profissional de saúde para auxiliá-los 54,5\% responderam que não e 45,5\% responderam sim. Apontam como estratégias de manejo emocional implementadas por eles mesmos durante a Pandemia: música (63,6\%), redes sociais $(54,5 \%)$, bate papo com amigos (45,5\%), jogos/games (36,4\%), igreja e namoro (27,3\%), esporte, escola, filmes e séries $(9,1 \%)$.

\section{Discussão}

O presente estudo identificou um conjunto de emoções negativas expressas por adolescentes escolares do ensino público no período de agosto/setembro 2020, transcorrido o primeiro semestre da Pandemia COVID-19 no Brasil. Em março iniciou-se o isolamento social como forma de conter/diminuir o avanço da doença. As aulas presenciais foram suspensas e todos os hábitos de vida foram modificados, ficando os adolescentes privados do convívio na Escola e demais espaços sociais.

Os dados coletados mostram que 66,6\% dos adolescentes escolares referiram cansaço ou desânimo, 45,5\% tristeza e 9,1\% depressão em decorrência do isolamento social promovido pela pandemia. As relações sociais e vínculos influenciam diretamente no estado emocional do adolescente. Interações positivas como amizade, carinho, amor transmitem segurança e, de maneira proporcionalmente inversa, as negativas como abandono, negligência, ausência acarretam danos à estrutura emocional.

$\mathrm{Na}$ ausência das aulas presenciais os alunos perderam um importante espaço de relações, bonito e cheio de vida, já que com ou sem as condições ideais de trabalho a escola pública tem o essencial conforme destaca Gadotti (2007): gente! E os dados deste estudo mostraram que os adolescentes escolares expressam a importância desse espaço para sua vida e felicidade, 45,5\% dos adolescentes informam que sentem muita falta do colégio e da convivência por ele propiciado.

Neste espaço os adolescentes constituem novas relações, interações e, sendo o homem um ser totalmente sociável, a interação com o outro é fundamental para formação e construção individual (Mello \& Teixeira, 2012). A escola promove encontros a partir dos "saberes socialmente construídos na prática comunitária", pode estimular discussões em que os alunos possam "assumir-se como ser social e histórico, como ser pensante, comunicante" criando uma identidade cultural (Freire, 2004).

O conhecimento cultural dos estudantes geralmente é visto como secundário, eles são tidas como pessoas que precisam ser ajudadas. Contudo, os escolares, durante o período da Pandemia, mesmo sem a ajuda da escolae atenção dos 
professores, sem o convívio com amigos e cuidados dos profissionais de saúde, foram capazes de adotar, por conta própria, estratégias para manejar suas emoções e promover sua saúde mental. Apresentando medidas de manejo das emoções indicadas pelos estudos da OMS (2020); Faro et al (2020); Schmidt et al (2020).

Freire (2004) ressalta que o ideal é que educandos e educadores trabalhem juntos para que a união dos saberes se torne "sabedoria" e que a partir da discussão da realidade criem a possibilidade de mudança. Ao criar espaços coletivos onde os alunos podem opinar, dar sugestões, a escola aborda e permite evidenciar e não inibir a compreensão de si mesmo dos adolescentes como sujeitos empoderados do seu próprio mundo.

Desenvolver o autoconhecimento é fundamental, pois na adolescência ocorrem muitas mudançascomo: aquisição de independência dos pais e família, tendência a questionar autoridades, desenvolvimento do sistema de valores e aquisição de identidade própria, estabelecimento de relações com pessoas da mesma idade. Tudo isso ocorre num curto período o que afeta diretamente seu estado emocional (Baptista, 2001)

\section{Conclusão e Implicações para a Prática Clínica}

Os resultados deste estudo revelam as repercussões do isolamento social decorrente da pandemia, principalmente no que diz respeito ao afastamento do espaço escolar, sobre a vivência emocional dos adolescentes escolares, indicando a ausência de suporte emocional oferecido pela escola ou serviços de saúde. Contudo, revela como um aspecto positivo, a capacidade dos adolescentes para o manejo de suas emoções e a adoção de estratégias adequadas para lidar com o estresse e a promoção da saúde mental, trazendo pistas para uma ação futura da equipe de atenção básica em saúde no âmbito do Programa Saúde na Escola.

Enquanto as medidas de isolamento se mantiverem, pretendemos utilizar uma ferramenta que possibilite trabalhar a promoção da saúde mental remotamente.

\section{Referências}

Baptista, M. N., Baptista, A. S. D. \& Dias, R. R. (2001). Estrutura e suporte familiar como fatores de risco na depressão de adolescentes. Psicol. cienc. prof. Brasília, 21(2), 52-61, June. https://doi.org/10.1590/S1414-98932001000200007.

Brasil. Ministério da Educação. (2020). Portaria n 343, de 17 de março de 2020. Dispõe sobre a substituição das aulas presenciais por aulas em meios digitais enquanto durar a situação de pandemia do Novo Coronavírus - COVID-19. https://www.in.gov.br/en/web/dou/-/portaria-n-343-de-17-de-marco-de-2020248564376.

Brooks, S. K., Webster, R. K., Smith, L. E., Woodland, L., Wessely, S., Greenberg, N. \& Rubin, G. J. (2020). The psychological impact of quarantine and how to reduce it: rapid review of the evidence. Lancet Infect Dis, 395(10227), 912-920. https://doi.org/10.1016/S0140-6736(20)30460-8.

Faro, A., Bahiano, M. A., Nakano, T. C., Reis, C., Silva, B. F. P. \& Vitti, L. S. (2020). COVID-19 e saúde mental: a emergência do cuidado. Estudos de Psicologia. Campinas, 37, e200074. Epub. June. https://doi.org/10.1590/1982-0275202037e200074.

Fundo das Nações Unidas para a Infância - UNICEF (2020). COVID-19: More than 95 per cent of children are out of school in Latin America and the Caribbean. Setembro. https://www.unicef.org/press-releases/covid-19-more-95-cent-children-are-outschool-latin-america-and-caribbean.

Freire, P. (2004). Pedagogia da autonomia: saberes necessários a prática educativa. Editora Paz e Terra. São Paulo.

Gadotti, M. (2007). A escola e o professor: Paulo Freire e a paixão de ensinar. Editora Publisher Brasil. São Paulo.

Junior, J. (2020). O papel fundamental da escola pública para enfrentar a COVID-19. A Gazeta, Publicado em 13/04/2020 https://www.agazeta.com.br/artigos/o-papel-fundamental-da-escola-publica-para-enfrentar-a-covid-19-0420.

Maia, B. R.; Dias, P. C. (2020). Ansiedade, depressão e estresse em estudantes universitários: o impacto da COVID-19. Estudos de Psicologia. Campinas, 37, e200067. https://doi.org/10.1590/1982-0275202037e200067.

Organização Mundial da Saúde - OMS e Organização Pan-Americana de Saúde - OPAS. (2020). Folha informativa COVID-19 - 2020. (Doença causada pelo novo coronavírus).

Pereira, A. S. et al. (2018). Metodologia da pesquisa científica. [e-book]. Santa Maria. Ed. UAB / NTE / UFSM. https://repositorio.ufsm.br/bitstream/handle/1/15824/Lic_Computacao_Metodologia-Pesquisa-Cientifica.pdf?sequence=1. 
Research, Society and Development, v. 10, n. 3, e47110313179, 2021

(CC BY 4.0) | ISSN 2525-3409 | DOI: http://dx.doi.org/10.33448/rsd-v10i3.13179

Schmidt, B.et al. (2020). Impactos na Saúde Mental e Intervenções Psicológicas Diante da Pandemia do Novo Coronavírus (COVID-19). SciELO Preprints, $1-26$. 